\title{
Plasma Neutrophil Gelatinase Associated Lipocalin (NGAL) - Early Biomarker for Acute Kidney Injury in Critically Ill Patients
}

\author{
Raluca Fodor ${ }^{1}$, Bianca Grigorescu2*, Mihaly Veres², Monica Orlandea², Judita Badea², \\ Katalin Hlavathy², Adrian Cioc ${ }^{2}$ \\ ${ }^{1}$ Department of Anaesthesiology and Intensive Care, University of Medicine and Pharmacy, Tirgu Mures, Romania \\ 2 Department of Anaesthesiology and Intensive Care, Emergency County Hospital, Tirgu Mures, Romania
}

\begin{abstract}
Introduction: NGAL (Neutrophil Gelatinase Associated Lipocalin) is a biomarker recently introduced into clinical practice for the early diagnosis of acute kidney injury (AKI). The aim of this study was to correlate the plasmatic NGAL value determined at admission with clinical progression and severity of AKI in critically ill patients.

Material and method: Thirty two consecutive critically ill adult patients at risk of developing AKI (trauma, sepsis), admitted in Intensive Care Unit of the Clinical County Emergency Hospital Mures, between January to March 2015 were enrolled in the study. For each patient included in the study plasma NGAL levels were determined on admission, and these were correlated with the degree of AKI development (according to AKIN criteria) at 48 hours and 5 days post admission. The discriminatory power of NGAL, creatinine, creatinine clearance and corrected creatinine (depending on water balance) were determined using the ROC (receiver-operating characteristic) and likelihood ratios.
\end{abstract}

Results: ROC curve analysis showed a better discriminatory capacity in terms of early diagnosis of AKI for NGAL ( $A \cup C=0.81$ for NGAL, AUC $=0.59$ for creatinine, $A \cup C=0.62$ for corrected creatinine, $A \cup C=0.29$ for creatinine clearance). The value of likelihood ratio was also significantly higher for NGAL (3.01 \pm 2.73 for NGAL, $1.27 \pm 1.14$ for creatinine, $1.78 \pm 1.81$ for corrected creatinine, and $0.48 \pm 0.33$ for creatinine clearance).

Conclusions: NGAL biomarker has a better discrimination capacity for early prediction of acute kidney injury compared to previously used markers.

Keywords: NGAL, AKI, corrected creatinine, critically ill patients

Received: 02 May 2015 / Accepted: 05 July 2015

\section{INTRODUCTION}

Acute renal failure (ARF) is frequently encountered in Intensive Care Units (ICU) associated with multiple organ failure, being a manifestation of acute renal injury. The progresses of 6-36\% of patients in ICUs develop complications involving ARF, and 50\% of the ARF cases are associated with sepsis [1]. The term "acute renal injury" (AKI) is preferred to "acute renal failure" as it better defines the type of renal impairment which can range from mild impairment of renal function to acute renal failure requiring renal substitution therapy.
Various biomarkers with increasing specificity have been used for the early diagnosis of acute myocardial infarction over the last fifty years. In contrast, detection methods for acute renal injury have remained relatively unchanged. Diagnosis relies, in the main, on the assessment of serum creatinine and oliguria levels, and these current methods of detection of acute renal injury delay diagnosis and treatment of disease for up to 48-72 hours [2].

New biomarkers of kidney damage are needed to facilitate and expedite kidney disease treatment. These

\footnotetext{
Correspondence to: Grigorescu Bianca, Department of Anesthesiology and Intensive Care, Emergency County Hospital Tirgu Mures, 50 Gheorghe Marinescu Street, Tirgu Mures, 540136, Romania. E-mail: biancagrigorescu20@yahoo.com

Fodor Raluca: Department of Anaesthesiology and Intensive Care, University of Medicine and Pharmacy, Tirgu Mures, Romania, Gh. Marinescu str., nr. 50, 540139, Romania Mihaly Veres: Department of Anaesthesiology and Intensive Care, Emergency County Hospital, Tirgu Mures, Romania, Gh. Marinescu str., nr.50, 540139, Romania

Monica Orlandea: Department of Anaesthesiology and Intensive Care, Emergency County Hospital, Tirgu Mures, Romania, Gh. Marinescu str., nr.50, 540139, Romania Judita Badea: Department of Anaesthesiology and Intensive Care, Emergency County Hospital, Tirgu Mures, Romania, Gh. Marinescu str., nr.50, 540139, Romania Katalin Hlavathy: Department of Anaesthesiology and Intensive Care, Emergency County Hospital, Tirgu Mures, Romania, Gh. Marinescu str., nr.50, 540139, Romania Adrian Cioc: Department of Anaesthesiology and Intensive Care, Emergency County Hospital, Tirgu Mures, Romania, Gh. Marinescu str., nr.50, 540139, Romania
} 
biomarkers should be able to detect early kidney damage, to identify patients at an increased risk of progressive disease [3], to guide the initiation of a therapy and to monitor the response to therapeutic interventions.

Neutrophil Gelatinase Associated Lipocalin (NGAL or $24 \mathrm{p} 3$, SIP24, lipocalin-2, orsiderocalin) [4], recently introduced in clinical practice, is a promising marker of early AKI, occurring shortly after renal injury and before an increase in serum creatinine. NGAL is a small molecule of $25-\mathrm{kDa}$ belonging to the superfamily of proteins called lipocalins [5]. As its name implies, this protein was initially described in neutrophils [6], where it exerts strong antibacterial properties and under specific conditions may promote cellular apoptosis [3]. NGAL is also normally expressed at very low levels in several human tissues, including kidneys, lungs, stomach, and colon. NGAL expression is markedly induced in injured epithelia [7].

NGAL also activates nephron formation in the embryonic kidney and possesses kidney-protective activ- between the 5th of January to 31st of March 2015. Exclusion criteria were kidney injury present at the time of admission and death within the first twenty four hours after ICU admission.

Blood samples were taken within the first six hours from admission to the ICU. NGAL was evaluated immediately after the blood samples were taken, using Alere Triage NGAL tests with the Alere Triage MeterPro device (Alere Inc., USA). Biochemical parameters including creatinine, were measured according to routine clinical laboratory methods. The creatinine clearance was assessed using the Cockcroft and Gault formula:

Creatinine Clearance $=\left[[140-\text { age }(y r)]^{*}\right.$ weight $\left.(\mathrm{kg})\right] /$ $\left[72^{*}\right.$ serum $\left.\mathrm{Cr}(\mathrm{mg} / \mathrm{dL})\right]$ (multiply by 0.85 for women).

Subsequently the degree of AKI development was assessed at 48 hours and 5 days after admission,using the AKIN criteria [9].

The following correction formula was used to obtain the relative volume expansion and to provide an estimate of plasma creatinine at an even fluid balance [10]:

$$
\text { Corrected } \mathrm{pCr}=\mathrm{pCr} * \frac{0.6 *(\text { Admission Weight })+\Sigma(\text { Daily Cumulative Fluid Balance })}{0.6 *(\text { Admission Weight })}
$$

ity [8]. Intraperitoneal or subcutaneous injection of recombinant NGAL after renal ischemia-reperfusion injury in mice, significantly improved renal function, if the injection was administered before the ischemia or one hour after reperfusion [8].

NGAL is synthesized systemically in response to kidney damage [3]. Increased regulation of NGAL expression is rapid, usually within 2 to 4 hours of injury. NGAL is also very stable and easily detected in urine [5].

The aim of this study was to correlate plasma NGAL value determined at admission with clinical progression and severity of AKI, in critically ill patients.

\section{MATERIAL AND METHOD}

The Institutional Review Board approval was obtained before enrolling the patients, and written informed consent obtained from all patients or legal guardians where needed. Thirty two patients were included in the study. The inclusion criteria were adult critically ill patients at risk of developing AKI due to trauma or sepsis, who had been admitted to the Intensive Care Unit of the Clinical County Emergency Hospital, Tîrgu Mureș,
The physiological and the disease-related variables necessary for calculating APACHE II (Acute Physiology and Chronic Health Evaluation) and SOFA (Sequential Organ Failure Assessment) scores, as tools for assessement the degree of desease severity, were also collected from patients' files on the first day of ICU admittance.

The therapeutic decision to administer fluids, vasopressors or diuretics was at the discretion of the physician in charge.

The data were processed using Microsoft Excell and SPSS statistics v.20 (Chicago, IL, USA). Data were expressed as mean $\pm \mathrm{SD}$. The significance level was set at $a=0.05$.

The discriminatory power of NGAL, creatinine, creatinine clearance and corrected creatinine levels were determined at 48 hours and 5 days after admission to the ICU, using the ROC (receiver-operating characteristic) and likelihood ratios for use as predictors of AKI.

\section{口ESULTS}

The present study enroled thirty two consecutive patients (18 males and 14 females). Patients' age ranged 
from $24-87$ years, the average being $56.7 \pm 17.7$ years. The main reasons for admission to the ICU were presence of sepsis (62.5\%) and trauma (37.5\%). The values for APACHE II and SOFA prognostic scores, on admission, are presented in Table 1.

Table 1. Prognostic scores at admission. Legend: APACHE II - Acute Physiology and Chronic Health Evaluation score; SOFA - The Sequential Organ Failure Assessment score

\begin{tabular}{lcccc} 
& Mean & $\begin{array}{c}\text { Standard } \\
\text { Deviation }\end{array}$ & Minimum & Maximum \\
APACHE II & 20.2 & 6.9 & 7.0 & 32.0 \\
\hline SOFA & 10.4 & 4.8 & 2.0 & 18.0 \\
\hline
\end{tabular}

According to AKIN criteria, of the 32 patients included in this study, 25\% developed AKI at 48 hours and $28 \%$ in 5 days.

The mean value of NGAL biomarker for the study group as a whole was $283 \pm 287 \mathrm{ng} / \mathrm{ml}$ (Figure 1). For the group of patients who did not develop AKI at 48 hours, it was $204 \pm 203 \mathrm{ng} / \mathrm{ml}$, and for the group of patients who did develop AKI at 48 hours, it was $521 \pm 376$ ng/ml (Figure 2).

The mean value of creatinine for the study group as a whole was $0.9 \pm 0.2 \mathrm{mg} / \mathrm{dl}$ (Figure 3), for the group of patients who did not develop AKI at 48 hours it was $0.88 \pm 0.2 \mathrm{mg} / \mathrm{dl}$, and for the group of patients who did develop AKI at 48 hours it was $0.96 \pm 0.28 \mathrm{mg} / \mathrm{dl}$. There was no statistically significant difference between the groups (p 0.05) (Figure 4).

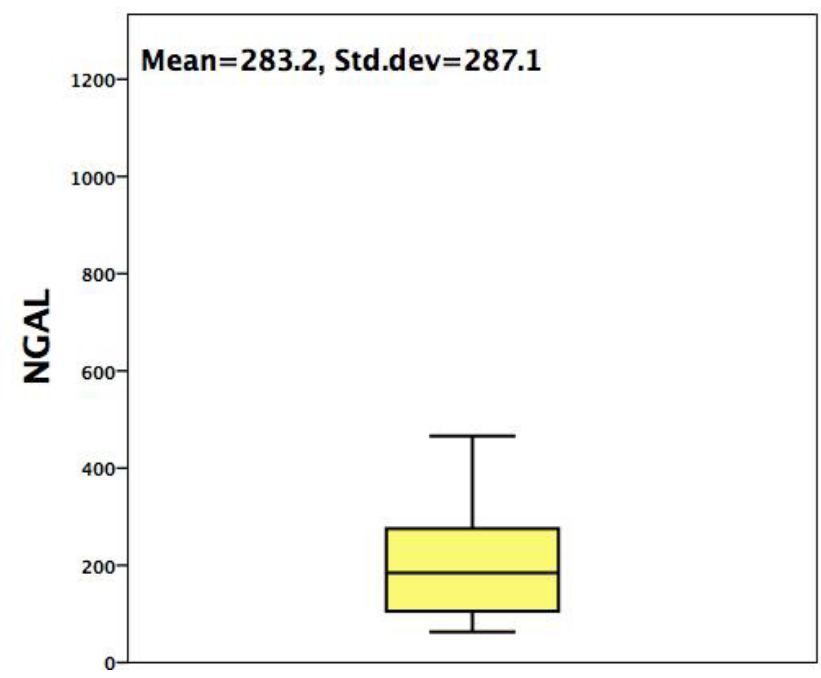

Figure 1. Mean value of NGAL at admission, for the entire study group

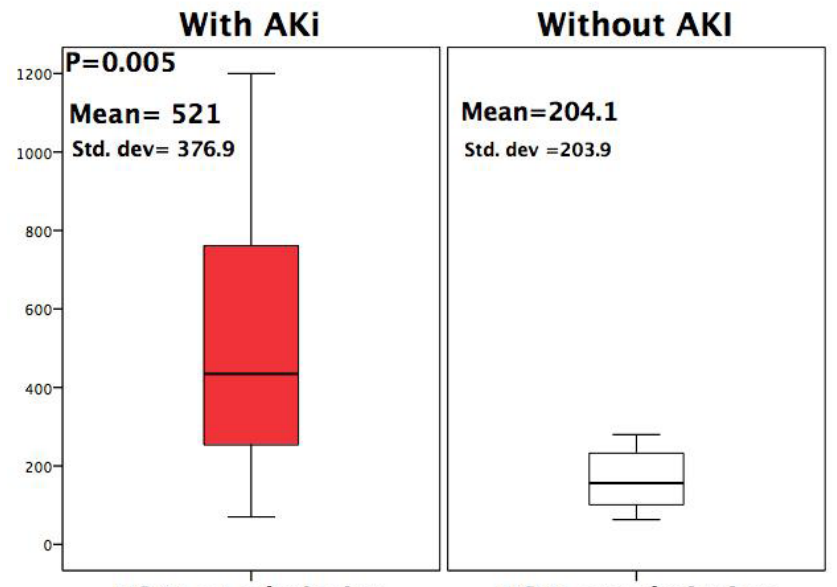

NGAL at admission

NGAL at admission

Figure 2. Mean values of NGAL at admission, for the patients who developed AKI at 48 hours compared to the patients who did not develop AKI

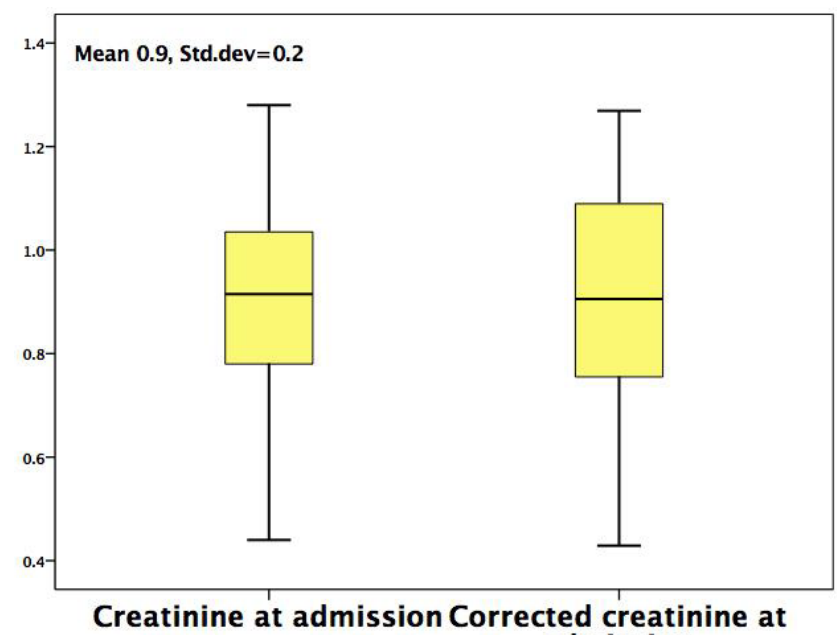
admission

Figure 3. Mean values of creatinine and corrected creatinine at admission, for theentire study group

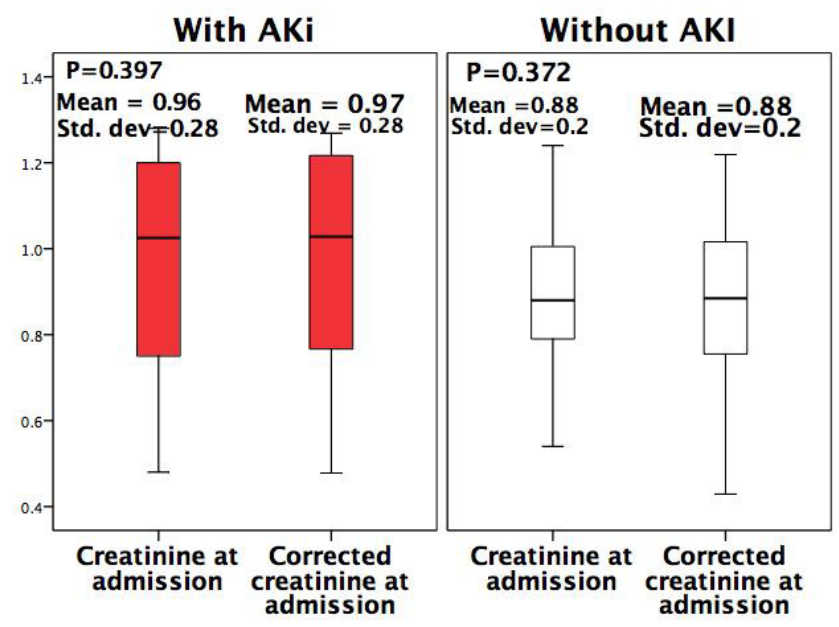

Figure 4. Mean values of creatinine and corrected creatinine at admission, for the patients who developed AKI at 48 hours compared to the patients who did not develop AKI 
The mean value of creatinine clearence for the study group as a whole was $101.3 \pm 47.7 \mathrm{ml} / \mathrm{min}$ (Figure 5), for the group of patients who did not develop AKI at 48 hours it was $109.41 \pm 50.4 \mathrm{ml} / \mathrm{min}$, and for the group of patients who did develop AKI at 48 hours it was 77.09 \pm $28.9 \mathrm{ml} / \mathrm{min}$ (Figure 6).

ROC curve analysis showed a better discriminatory capacity for NGAL, in terms of early diagnosis of AKI both at 48 hours, the Area under curve (AUC) being 0.81 for NGAL, 0.59 for creatinine, 0.62 for corrected

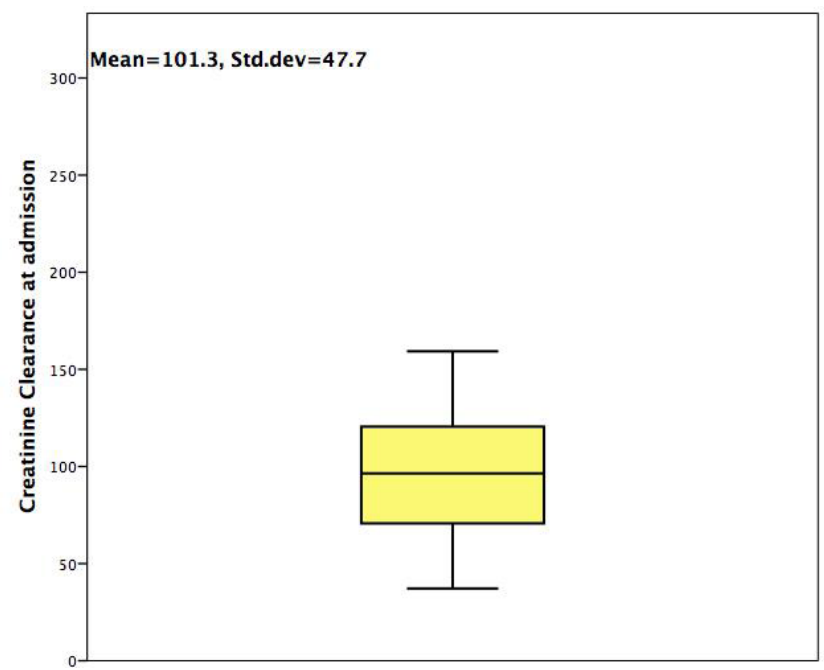

Figure 5. Mean value of creatinine clearance at admission, for theentire study group

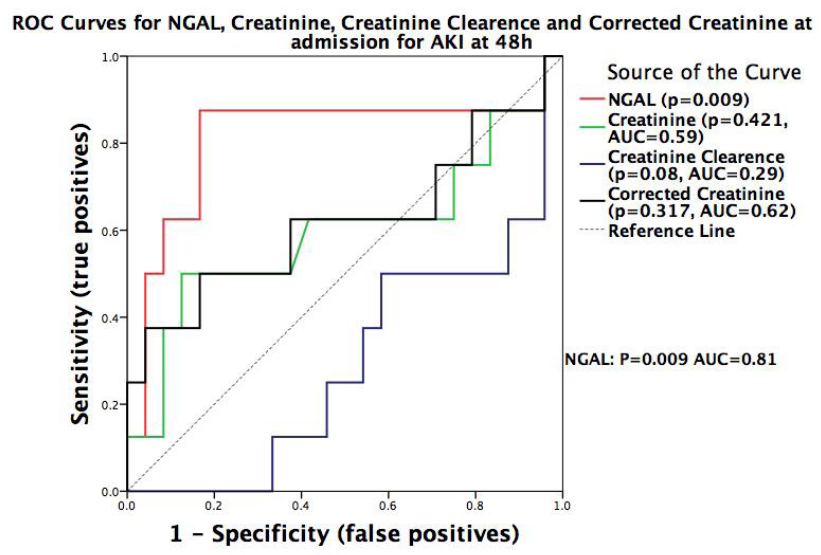

Figure 7. Receiver-operating characteristic curves for NGAL, creatinine, creatinine clearance and corrected creatinine at admission for AKI at $\mathbf{4 8}$ hours. ROC curve analysis showed a better discriminatory capacity for NGAL compared to previous used markers: $A U C=0.81$ for NGAL, AUC $=0.59$ for creatinine, $A U C=0.62$ for corrected creatinine, $A U C=0.29$ for creatinine clearance creatinine, and 0.29 for creatinine clearance) (Figure 7). At 5 days AUC was 0.85 for NGAL, 0.62 for creatinine, 0.62 for corrected creatinine, and 0.18 for creatinine clearance (Figure 8). At both 48 hours and 5 days only NGAL was significantly different.

The correlation coefficient methods of Youdenand Matthews, were used to calculate the cutoff value for the positive diagnosis of AKI at forty eight hours. Both index values may range between 0 and 1 . The NGAL value which determines the highest index values (clos-

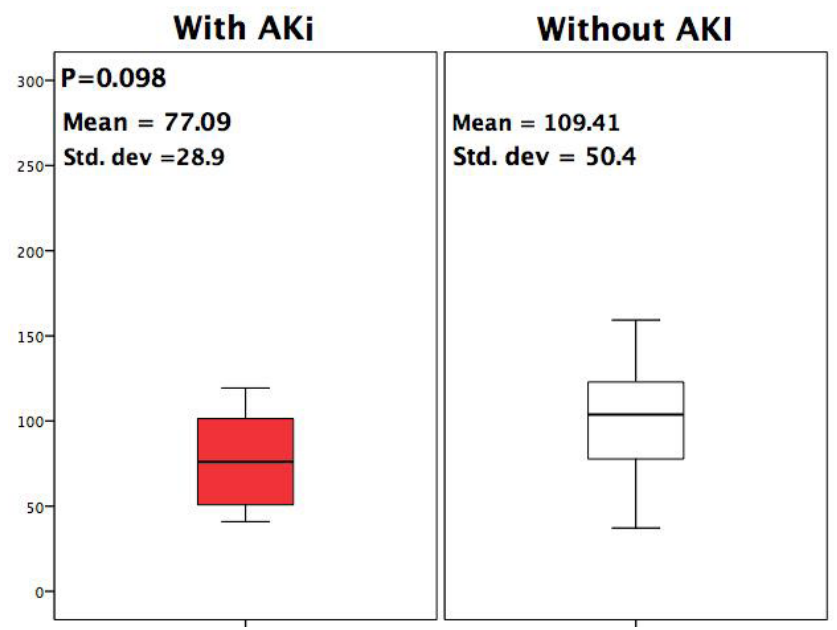

Figure 6. Mean values of creatinine clearance at admission, for the patients who developed AKI at $\mathbf{4 8}$ hours compared to the patients who did not develop AKI

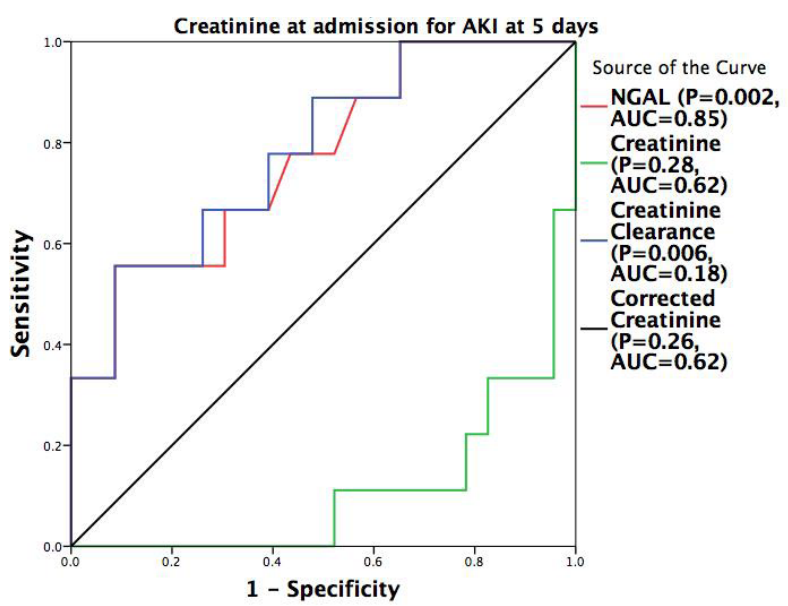

Figure 8. Receiver-operating characteristic curves for NGAL, creatinine, creatinine clearance and corrected creatinine at admission for AKI at 5 days. ROC curve analysis showed a better discriminatory capacity for NGAL compared to previous used markers: $A U C=0.85$ for NGAL, AUC $=0.62$ for creatinine, $A U C=0.62$ for corrected creatinine, $A \cup C=0.18$ for creatinine clearance 
est to 1 ) is chosen as cutoff point. The calculated cutoff value using these two methods was $252 \mathrm{ng} / \mathrm{ml}$, with a sensitivity of $87 \%$ and a specificity of $83 \%$. The study had an $87 \%$ power to discern between true positive results (true positive rate) and the false positive results of $83 \%$ (false positive rate) (Figure 9, 10).

The value of likelihood ratio was also higher for NGAL (3.01 \pm 2.73 for NGAL, $1.27 \pm 1.14$ for creatinine, $1.78 \pm 1.81$ for corrected creatinine, and $0.48 \pm 0.33$ for creatinine clearance) (Table 2).

Table 2. Positive likelihood ratios at admission for AKI at 48 hours. The likelihood ratio evaluates the probability of developing AKI in patients with a value of NGAL higher than the cutoff $(252 \mathrm{ng} / \mathrm{ml})$. The value of likelihood ratio was also significantly higher for NGAL

\begin{tabular}{lcccc} 
& Mean & $\begin{array}{c}\text { Standard } \\
\text { Deviation }\end{array}$ & $\begin{array}{c}\text { Mini- } \\
\text { mum }\end{array}$ & $\begin{array}{c}\text { Maxi- } \\
\text { mum }\end{array}$ \\
\hline LR NGAL & 3.01 & 2.73 & 0.00 & 11.91 \\
\hline $\begin{array}{l}\text { LR Creatinine } \\
\text { Clearance }\end{array}$ & 0.48 & 0.33 & 0.00 & 1.04 \\
\hline LR Creatinine & 1.27 & 1.14 & 0.00 & 4.52 \\
\hline $\begin{array}{l}\text { LR Corrected } \\
\text { Creatinine }\end{array}$ & 1.78 & 1.81 & 0.00 & 9.00 \\
\hline
\end{tabular}

The efficiency of NGAL as a predictive marker for AKI was determined using a logistic regression model, comparing the NGAL values at admission to the likelihood of developing AKI at forty eight hours. The statis-

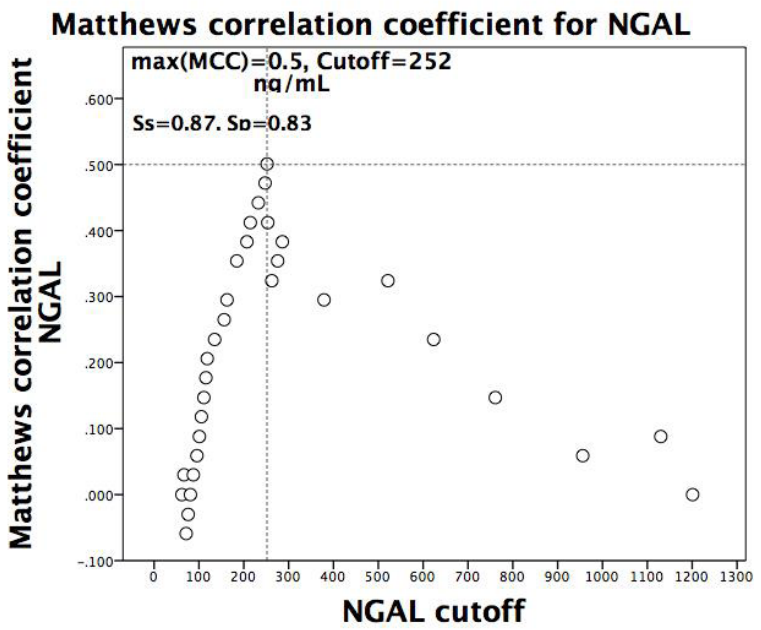

Figure 9. Mathews correlation coefficient for NGAL. The NGAL value which determines the highest index values (closest to 1 ) is chosen as cutoff point. The calculated cutoff value was $252 \mathrm{ng} / \mathrm{ml}$, with a sensitivity of $87 \%$ and a specificity of $83 \%$.Legend: Ss - sensibility, Sp - specificity. tical model obtained has a precision of $81.3 \%(\mathrm{p}=0.027)$ in discriminating patients who have developed AKI depending on the NGAL value (Figure 11).

The Hosmer-Lemeshow test was used to verify the precision of the logistic regression model. Analyzing the charts, an almost identical overlap of the power of prediction with the cases which had positive NGAL results and developed AKI ( $\mathrm{p}=0.419)$ was observed (Figure 12).

\section{DISCUSSION}

The current clinical practice is to use serum creatinine levels as a marker of kidney function inspite of it being a relatively poor indicator of the early stages of AKI. Serum creatinine may also vary with age, gender, muscle mass, medications and hydration status [11] and additionally, serum creatinine levels may stay unchanged until aproximately $50 \%$ of kidney function is lost [2]. Under the AKIN criteria, diagnosis of AKI is categorized as an abrupt (within 48 hours) reduction in kidney function defined as an absolute increase in serum creatinine $>0.3 \mathrm{mg} / \mathrm{dL}$, a percentage increase in serum creatinine of $>50 \%$ or a reduction in urine output to $<0.5 \mathrm{ml} / \mathrm{kg}$ per hour for $>6$ hours [9]. The dilution of serum after volume expansion may be a primary factor in limiting the ability of plasmatic creatinine changes to diagnose AKI [10]. Consequently, a recent proposed correction formula for relative volume expansion was used.

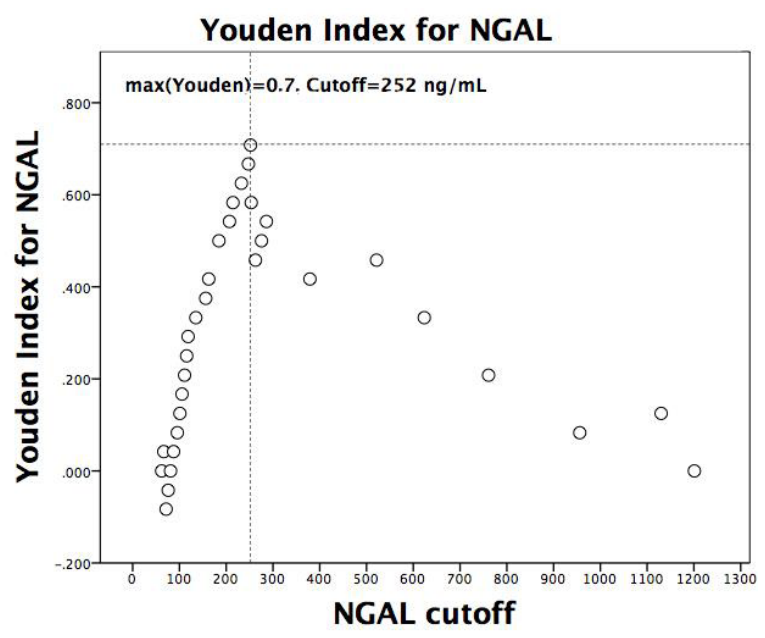

Figure 10. Youden Index for NGAL. The NGAL value which determines the highest index values (closest to 1 ) is chosen as cutoff point. The calculated cutoff value was 252 $\mathrm{ng} / \mathrm{ml}$, with a sensitivity of $87 \%$ and a specificity of $83 \%$. Legend: Ss - sensibility, Sp- specificity. 
Available online at: www.jccm.ro

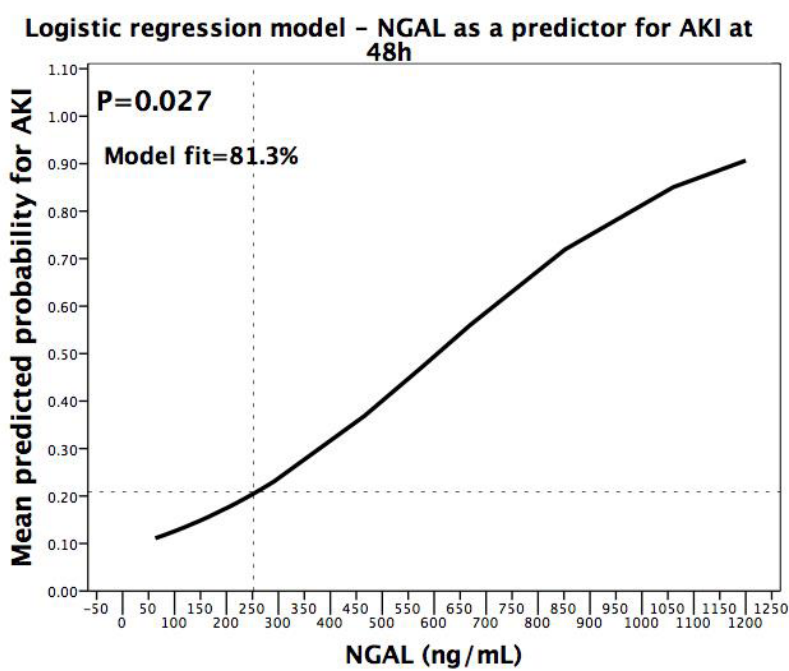

Figure 11. Logistic regression model - NGAL as a predictor for AKI at $\mathbf{4 8}$ hours. We compared the NGAL values at admission to the likelihood of developing AKI at 48 hours. The statistical model obtained has a precision of 81.3\% ( $p=0.027)$ in discriminating patients who have developed AKI depending on the NGAL value. The risk of developing AKI at the cutoff value is of $20 \%$. This risk increases exponentially as the cutoff value is exceeded.

Early detection of kidney damage in critically ill patients contributes significantly to patient managment and clinical outcomes [2]. Recent studies suggest that NGAL has the potential to be an ideal biomarker of early kidney damage in patients at risk [3,5,11], particularly when the timing of the renal insult is known [2].

There are numerous studies that highlight the effectivness of NGAL in the early diagnosis of acute renal failure in patients after cardiopulmonary bypass. It was demonstrated that plasma NGAL becomes positive as early as 1-2 hours after cardio-pulmonary bypass $[12,13]$.

Plasma and urine NGAL measurements were also found to be predictive biomarkers of AKI following contrast administration $[14,15]$

NGAL was found to be an excellent biomarker for the early diagnosis of AKI in children and adults undergoing renal transplantation [16]. Biopsies of kidneys obtained one hour after vascular anastomosis revealed a significant correlation between NGAL staining intensity and the subsequent development of delayed graft function [17].

NGAL proved to be a major marker for monitoring the progression of chronic kidney diseases, such as polycystic kidney disease, glomerulonephritis or vasculitis. Serum concentrations of NGAL increase before an increase in serum creatinine levels [5,18-20].
The Journal of Critical Care Medicine 2015;1(4) • 159

Hosmer and Lemeshow test for model fit

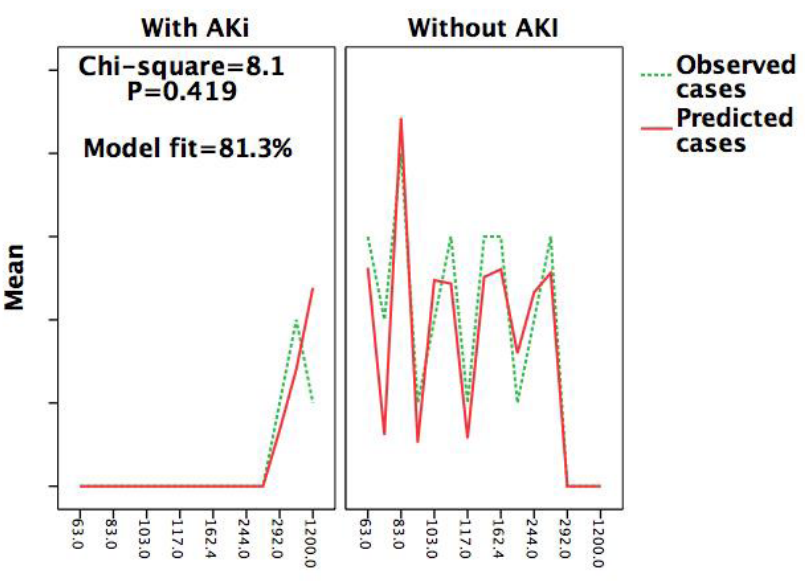

Figure 12. Hosmer and Lemeshow test for model fit.This test verifies the precison of the model by comparing the power of prediction with the cases which had positive NGAL values and developed AKI. Analyzing the charts, an almost identical overlap of the power of prediction with the cases which had positive NGAL results and developed AKI $(p=0.419)$ was observed.

In the present study, we found that mean plasma NGAL level was significantly higher in critically ill patients who developed AKI at forty eight hours compared to patients who did not develop AKI at forty eight hours after admission ( $\mathrm{p}=0.005)$. NGAL proved to be a good predictor for the development of AKI within the 48 hours following the first measurement, with an AUC - ROC of 0.81. The data from the present study is in agreement with those of Cruzet al [2], who reported that plasma NGAL confirmed the diagnosis of AKI up to 48 hours prior to a clinical diagnosis based on AKI definitions. It was also reported that NGAL levels were significantly elevated in ICU patients with severe illness compared to healthy adults and increased in AKI patients compared to non-AKI patients [2].

In contrast, a recent study [21] found that plasma NGAL and also proadrenomedullin and cystatin C performed well, but did not significantly outperform serum creatinine in identifying oliguric patients with poor renal outcome. The non-superiority of AKI biomarkers over serum creatinine levels, as evidenced in this study [21] might be explained by the focus on the oliguric patients.

In the present study the best cut-off value to predict early stage AKI was determined. For plasma NGAL this value was $252 \mathrm{ng} / \mathrm{ml}$, with a sensitivity of $87 \%$ and a specificity of $83 \%$. Previous studies gave the best cut-off 
level for NGAL as $435 \mathrm{ng} / \mathrm{ml}$ in patients with chronic kidney diseases [22].

To emphasize the accuracy of the diagnostic test, a "likelihood ratio" was performed. This ratio evaluates the probability of developing AKI in patients with a value of NGAL higher than the cutoff $(252 \mathrm{ng} / \mathrm{ml})$. Generally, a value for this ratio higher than 2 is considered acceptable and a value over 10 being excellent. The value of likelihood ratio was also highest for NGAL. The risk of developing AKI at the cutoff value, according to the logistic regression model, comparing the NGAL values at admission to the likelihood of developing AKI at forty eight hours, is $20 \%$. This risk increases exponentially as the cutoff value is exceeded.

The Hosmer-Lemeshow test verifies the precision of the logistic regression model by comparing the power of prediction with the cases which had positive NGAL values and developed AKI. In this test, the $\mathrm{p}$ value must be $>0.05$ for the test to validate the power of prediction of the model.

NGAL increases independently from the setting of AKI, so it can be applied to different patients with AKI though it is not suitable as an indicator of the etiology of AKI [23]. A combination of biomarkers may be necessary to provide the best diagnostic and prognostic information in a context-specific manner [24].

The weak point of NGAL is that it is not kidney specific, thus confounding the predictive ability of plasma NGAL for AKI $[2,25]$. Its levels may increase in other pathological conditions. Elevated plasma levels of NGAL were also found as an expression of colorectal neoplasia and inflammatory bowel diseases. High concentrations of NGAL were observed in colonic epithelium from inflamed areas such as diverticulitis, inflammatory bowel disease or appendicitis and also in premalignant or malignant neoplastic lesions of the colon [26].

We acknowledge that the enrolled numbers in the present study are small and a study with larger numbers is being considered.

\section{- CONCLUSIONS}

NGAL biomarker has a better discrimination capability for early prediction of acute kidney injury in critically ill patients compared to previously used markers. The use of this novel biomarker may allow early targeted therapy for critically ill patients, initiated at a time when the protection of stressed renal tissue is still possible.

\section{AKNOWLEDGEMENT}

This paper was published under the frame of European Social Found, Human Resources Development Operational Programme 2007-2013, project no. POSDRU/159/1.5/S/136893"

\section{DEFERENCES}

1. Singbartl K, Kellum JA. AKI in the ICU: Definition, Epidemiology, Risk Stratification, and Outcomes. Kidney Int. 2012;81(9):81925.

2. Cruz DN, Cal M, Garzotto M. Plasma neutrophil gelatinaseassociated lipocalin is an early biomarker for acute kidney injury in an adult ICU population. Intensive Care Med. 2010; $36: 444-51$.

3. Fatemeh G, Faranak K, Esmat G, Rahimipour A, Houshang A, Malihe N. Assessment of Neutrophil gelatinase-associated lipocalin (NGAL) as an early biomarker for detection of renal impairment in hypertensive patients. Journal of Paramedical Sciences. 2014;5:63-6.

4. Flower DR, North AC, Sansom CE. The lipocalin protein family: structural and sequence overview. Biochim Biophys Acta. 2000;1482:9-24.

5. Bolignano D, Donato V, Coppolino G, et al. Neutrophil Gelatinase-Associated Lipocalin (NGAL) as a Marker of Kidney Damage.American Journal of Kidney Diseases. 2008;52:595605.

6. Kjeldsen $L$, Johnsen $A H$, Sengelov $H$, Borregaard N. Isolation and primary structure of NGAL, a novel protein associated with human neutrophil gelatinase. J Biol Chem. 1993;268:10425-32.

7. Devarajan P: Emerging Biomarkers of Acute Kidney Injury. In Ronco C, Bellomo R, Kellum JA: Acute Kidney Injury. ContribNephrol, vol 156. Basel:Karger. 2007, pp 203-12.

8. K Mori, K Nakao. Neutrophil gelatinase-associated lipocalin as the real-time indicator of active kidney damage. Kidney International. 2007;71:967-70.

9. Mehta RL, Kellum JA, Shah SV, et al. Acute Kidney Injury Network. Acute Kidney Injury Network: report of an initiative to improve outcomes in acute kidney injury. Crit Care. 2007;11:R31.

10. Prowle JR, Leitch A, Kirwan CJ, Forni LG. Positive fluid balance and AKI diagnosis: assessing the extent and duration of 'creatinine dilution'. Intensive Care Med. 2015;41:160-1.

11. Devarajan P. Neutrophil gelatinase-associated lipocalin (NGAL): A new marker of kidney disease. Scand J Clin Lab Invest Suppl. 2008;241:89-94.

12. Perry TE, Muehlschlegel JD, Liu KY. Plasma Neutrophil Gelatinase-Associated Lipocalin and Acute Postoperative Kidney Injury in Adult Cardiac Surgical Patients. AnesthAnalg. 
Available online at: www.jccm.ro

\section{0;110:1541-7.}

13. Fielitz AH, Bellomo R, Devarajan P, et al. The predictive performance of plasma neutrophil gelatinase-associated lipocalin (NGAL) increases with grade of acute kidney injury. Nephrol Dial Transplant. 2009;24:3349-54.

14. Bachorzewska GH, Malyszko J, Sitniewska E, Malyszko JS, Dobrzycki S. Neutrophil gelatinase-associated lipocalin and renal function after percutaneous coronary interventions. Am J Nephrol. 2006;26:287-92.

15. Malyszko J, Bachorzewska GH, Malyszko JS, Dobrzycki S. Could NGAL predict renal function after percutaneous coronary interventions? J Am SocNephrol. 2006;17:406A.

16. Parikh CR, Jani A, Mishra J et al. Urine NGAL and IL-18 are predictive biomarkers for delayed graft function following kidney transplantation. Am J Transplant. 2006;6:1639-45.

17. Mishra J, Ma Q, Kelly $C$, et al. Kidney NGAL is a novel early marker of acute injury following transplantation. Pediatr Nephrol. 2006;21:856-63.

18. Mitsnefes M, Kathman T, Mishra J, et al. Serum NGAL as a marker of renal function in children with chronic kidney disease. Pediatr Nephrol. 2007;22:101-8.

19. Bolignano D, Coppolino G, Campo S, et al. Urinary neutrophil gelatinase-associated lipocalin (NGAL) is associated with severity of renal disease in proteinuric patients. Nephrol Dial Transpl. 2008;23:414-6.
The Journal of Critical Care Medicine 2015;1(4) • 161

20. Bolignano D, Coppolino G, Campo S, et al. Neutrophil gelatinase associated lipocalin in patients with autosomal-dominant polycystic kidney disease. Am J Nephrol. 2007;27:373-8.

21. Legrand $M$, Jacquemod $A$, Gayat E. Failure of renal biomarkers to predict worsening renal function in high-risk patients presenting with oliguria. Intensive Care Med. 2015;41:68-76.

22. Bolignano D, Lacquaniti A, Coppolino G, et al. Neutrophil gelatinase-associated lipocalin (NGAL) and progression of chronic kidney disease. Clinical Journal of the American Society of Nephrology. 2009;4:337-44.

23. Haase M, Bellomo R, Fielitz AH. Neutrophil gelatinaseassociated lipocalin: a superior biomarker for detection of subclinical acute kidney injury and poor prognosis. Biomarkers Med. 2011;5(4):415-7.

24. Devarajan P. Biomarkers for the early detection of acute kidney injury. Current Opinion in Pediatrics. 2011;23:194-200.

25. Poniatowski B, Malyszko J, Bachorzewska GH, Malyszko JS, Dobrzycki S. Serum neutrophil gelatinase-associated lipocalin as a marker of renal function in patients with chronic heart failure and coronary artery disease. Kidney Blood Press Res. 2009;32:77-80.

26. Nielsen BS, Borregaard N, Bundgaard JR, Timshel S, Sehested $M$, Kjeldsen L. Induction of NGAL synthesis in epithelial cells of human colorectal neoplasia and inflammatory bowel diseases. Gut. 1996;38:414-20. 